\title{
Polarization of $\Lambda$ Hyperons In Proton-Proton Reactions At 3.5 GeV Measured With Hades
}

\section{Rafal Lalik*Technische Universität München Fakultät für Physik, E62}

E-mail: rafal.lalikeph.tum.de

for the HADES Collaboration

First results of the $\Lambda$ polarization measured by HADES in proton-proton reactions at $\sqrt{s}=$ $3.18 \mathrm{GeV}$ are reported. According to theory and a measurement of the $\Lambda$ polarization at the same beam kinetic energy in $\mathrm{p}+\mathrm{Nb}$ reaction, the polarization is expected to have small and negative value. The present results do not yet allow to draw conclusions on the polarization in $\mathrm{p}+\mathrm{p}$ reactions. In the analysis we experience large uncertainty in the hyperon direction and positive but small polarization in the spin-aligned direction.

The 26th International Nuclear Physics Conference

11-16 September, 2016

Adelaide, Australia

${ }^{*}$ Speaker. 


\section{Introduction}

Although unexpected by theory, the hyperon polarization was measured in variety of hadron beams, energies and targets sizes (experimental Refs. [1-3], theory and review Refs. [4-6]). To review these measurements we study the charged particles decay of $\Lambda \rightarrow \mathrm{p} \pi^{-}$, as this is accessible in a particle detector. The direction of the spin of the initial nucleons is conserved in the strong interactions leading to the production of $\Lambda$, having its spin oriented along the normal vector of the $\Lambda$ production plane. As a direct consequence of the parity-violating nature of the weak decay $\Lambda \rightarrow \mathrm{N} \pi$, the $\Lambda$ decays via an angular anisotropy of the daughter nucleon in respect to the spin of the mother $\Lambda$ hyperon. This makes the $\Lambda$ hyperon a self-analyzing particle.

In this paper we present very first results from the $\Lambda$ polarization analysis in $p+p$ reaction, specifically the experimental data and reference simulations with the reconstruction performance studies. Results are simultaneously evaluated in the three polarization angles $\zeta_{i}$, where $i \in\{x, y, z\}$, which are commonly defined as:

$$
\cos \zeta_{i}=\frac{\overrightarrow{\mathrm{p}^{\prime}} \cdot \vec{n}_{i}}{\left|\overrightarrow{\mathrm{p}^{\prime}}\right|\left|\vec{n}_{i}\right|}
$$

where $\overrightarrow{\mathrm{p}^{\prime}}$ is the proton momentum vector in the $\Lambda$ rest frame, and $n_{i}$ are three orthogonal vectors defined in the (b)eam- $\Lambda$ production plane:

$$
\vec{n}_{x}=\frac{\vec{b} \times \vec{\Lambda}}{|\vec{b} \times \vec{\Lambda}|}, \quad \vec{n}_{y}=\vec{n}_{z} \times \vec{n}_{x}, \quad \vec{n}_{z}=\frac{\vec{\Lambda}}{|\vec{\Lambda}|} .
$$

Having a distribution of $\Lambda$ as a function of $\cos \zeta_{i}$, the polarization can be derivied in a various ways. In this paper we consider (a) a linear fit to the $\Lambda$ yield:

$$
\frac{\mathrm{d} N}{\mathrm{~d} \cos \zeta_{i}}=A\left(1+\alpha \mathscr{P}_{i} \cos \zeta_{i}\right)
$$

and (b) asymmetry of integrals between up $\left(U, U: \cos \zeta_{i}>0\right)$ and down $\left(D, D: \cos \zeta_{i}<0\right)$ oriented cosines:

$$
\mathscr{P}_{i}^{\mathrm{ud}}=\frac{2}{\alpha} \frac{U-D}{U+D}
$$

where $\alpha=0.642 \pm 0.013$ is the analyzing power of the parity-violating weak decay [7], and $A$ is a scaling factor. However, due to parity conservation in strong interaction, no polarization is expected in $y$ and $z$-direction, d.h. parallel to the production plane.

According to Refs. $[4,5]$, the polarization at low $p_{\mathrm{t}}$ values lower than $1 \mathrm{GeV} / \mathrm{c}$ is expected to be on order of -0.1 . It has been confirmed in HADES by measurements of $\mathrm{p}+\mathrm{Nb} \rightarrow \Lambda+X$ reaction [8] which showed an average value of $-0.119 \pm 0.005 \pm 0.016$ over different extraction methods.

\section{Experiment}

The data were obtain with the High Acceptance Dielectron Spectrometer (HADES) at the heavy-ion synchrotron SIS18 in GSI Helmholtzzentrum für Schwerionenforschung in Darmstadt. The spectrometer is a charged-particles detector with hexagonal geometry. The toroidal magnet 
centered at the beam axis is surrounded by two frontal and two rear layers of the multiwire drift chamber (MDC) detectors. The fixed target is surrounded by the ring imaging Cherenkov (RICH) detector, which is directly followed by the MDC detectors. The time of flight scintillator detectors TOF and TOFino follow the last MDC layer and provide time measurement. The last detector in the spectrometer is the PreShower detector for additional electron identification. System of TOF, TOFino and PreShower detectors constitutes a multiplicity electron and trigger array (META). Additional single forward wall detector centered at the beam axis and located $7 \mathrm{~m}$ behind the target is used for measurements of spectators in nuclei and heavy nuclei experiments. Position of the MDC detectors determines polar angle coverage of $18^{\circ}$ to $85^{\circ}$ and $85 \%$ in the azimuthal angle. The momentum resolution for charged particles spans from $1 \%$ to $4 \%$. The full description of the HADES spectrometer is in Ref. [9]. The particle identification (PID) is performed using energy deposition in the MDC and reconstructed tracks bending in the magnet (field strength from $0.9 \mathrm{~T}$ to 3.6 T). Missing of the diamond start detector positioned directly in the front of the target prevents effective PID reconstruction with the use of time of flight detectors.

Data for the analysis were taken in 2007 with the proton beam of about $10^{6}$ particles/s inciding on the liquid hydrogen target of $50 \mathrm{~mm}$ thickness, corresponding to $0.7 \%$ interaction rate. The LVL1 trigger selection in the META system required at least three charged particles detected. In total, $1.14 \times 10^{9}$ events have been collected. Additional $1.2 \times 10^{5}$ calibration events with an empty target configuration for studies of off-target background reactions have been measured.

$\Lambda$ hyperons were reconstructed by investigating charged particle's decay channel $\Lambda \rightarrow \mathrm{p} \pi^{-}$ (branching ratio of $63 \%$ ). Protons and pions were selected by 2-dimensional cuts in the energy deposition versus charge $\times$ momentum distribution. Reconstructed four-vectors for each pair of identified protons and pions were combined and resulted in the mother $\Lambda$ candidate. The $\Lambda$ decay vertex (secondary vertex $[\mathrm{SV}]$ ) was associated to the middle of the distance of the closest approach (DCA) between two daughter tracks. All pairs where DCA was larger than $10 \mathrm{~mm}$ were assumed as uncorrelated and rejected. The primary vertex (PV) was reconstructed by intersecting the $\Lambda$ candidate track with all other reconstructed tracks, or if not available, with an average beam position estimated for that event. Only the events where secondary vertex followed the primary vertex $\left(z_{\mathrm{SV}}>z_{\mathrm{PV}}\right.$ were accepted. Additionally, the angle between the $\Lambda$ candidate and the vector connecting the primary and the secondary vertex had to be smaller than $0.1 \mathrm{rad}$, and the primary vertex had to be allocated inside the target region in the $z$-range of $-50 \mathrm{~mm}$ to $-30 \mathrm{~mm}$ while target covered $-65 \mathrm{~mm}$ to $-15 \mathrm{~mm}$ range. The $15 \mathrm{~mm}$ separation from the liquid hydrogen target ends was adopted to remove contributions of off-target reaction in the kapton windows studied in the empty target data. Additional cut on the missing mass lower than $1400 \mathrm{MeV} / \mathrm{c}^{2}$ was applied to reduce remaining background of off-target reactions.

The resulting invariant mass spectrum has been fit with double gaussian function for the $\Lambda$ peak and polynomial of the fifth order for the background description. In total $(252.8 \pm 1.2) \times 10^{3}$ $\Lambda$ s have been reconstructed with the mean mass of $1115.122 \pm 0.009 \mathrm{MeV} / \mathrm{c}^{2}$ and signal to background ratio of 0.47 .

Cosines of the three polarization angles $\zeta_{i}$ ( -1 to 1 ) were divided into 20 intervals of 0.1 width each and an individual invariant mass spectrum for each interval was separately fit in order to calculate $\Lambda$ yield in each of them. The normalized distributions of $\Lambda$ yields are shown in Fig. 1 as red inverted triangles. Shapes of the distribution are purely related to the acceptance of the detector. 


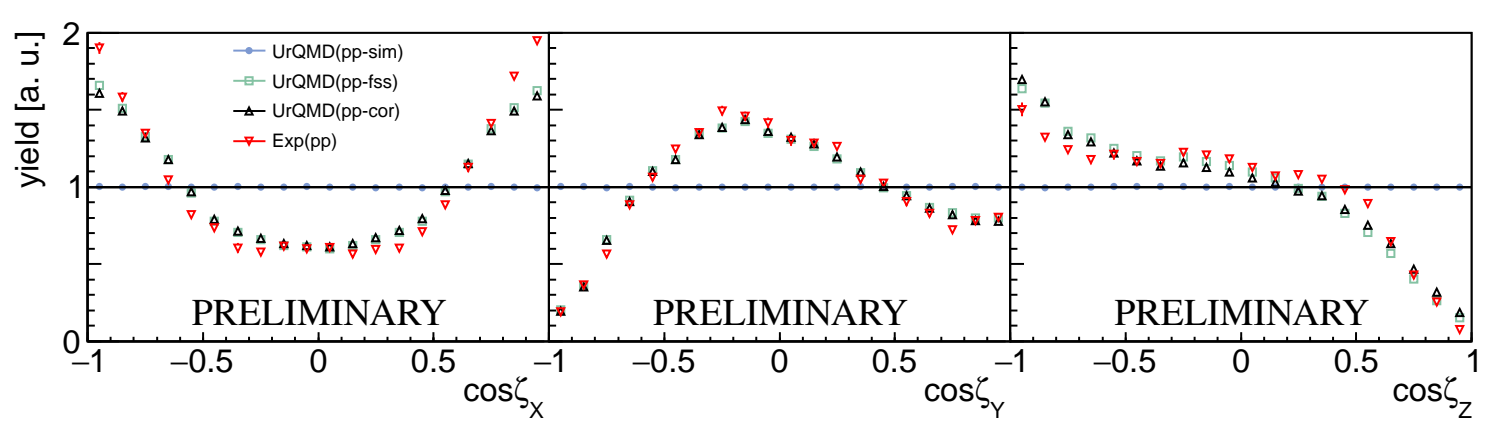

Figure 1: $\Lambda$ yields for the experimental data (red inverted triangles) and UrQMD simulations before (blue circles) and after (green squares) HADES acceptance filtering and UrQMD correction matrix (black triangles). The yields are normalized to 2 .

\section{Simulations and results}

UrQMD [10] simulation has been used to generate a sample of polarization-free (see blue circles in Fig. 1) $\Lambda$ decays and study acceptance of the detector and prepare correction matrices. The generated sample was filtered for the HADES detector acceptance and a full reconstruction process was performed as described for the experimental data. The resulting distribution of cosines is represented by the green squares in Fig. 1. Since the input does not carry any polarization, the resulting correction matrix defined as a ratio between filtered and simulated sample is nearly overlapping (statistical fluctuations in the simulation determine small deflection from the filtered signal) with the filtered sample (black triangles in Fig. 1).

Figure 2 shows the experimental yields (black triangles) corrected for the spectrometer acceptance (red inverted triangles). Red dashed line presents the linear fit as introduced in Eq. (1.3). $P_{\text {fit }}$ is the polarization extracted from the fit, $P_{\mathrm{ud}}$ is the polarization extracted from the U-D assymetry (Eq. (1.4)), and $U$ and $D$ are respective integrals. It can be observed that there is no measurable polarization in the $\zeta_{x}$ and $\zeta_{y}$ angles, but there seems to be significant slope in the range of -1 to 0.5 in the cosine of the $\zeta_{z}$ angle. Cosines above 0.5 are known to be sensitive to the acceptance effects in the HADES simulations and therefore this region is not reliable to draw any conclusions.

To study influence of the reconstruction quality on the polarization angle determination, each

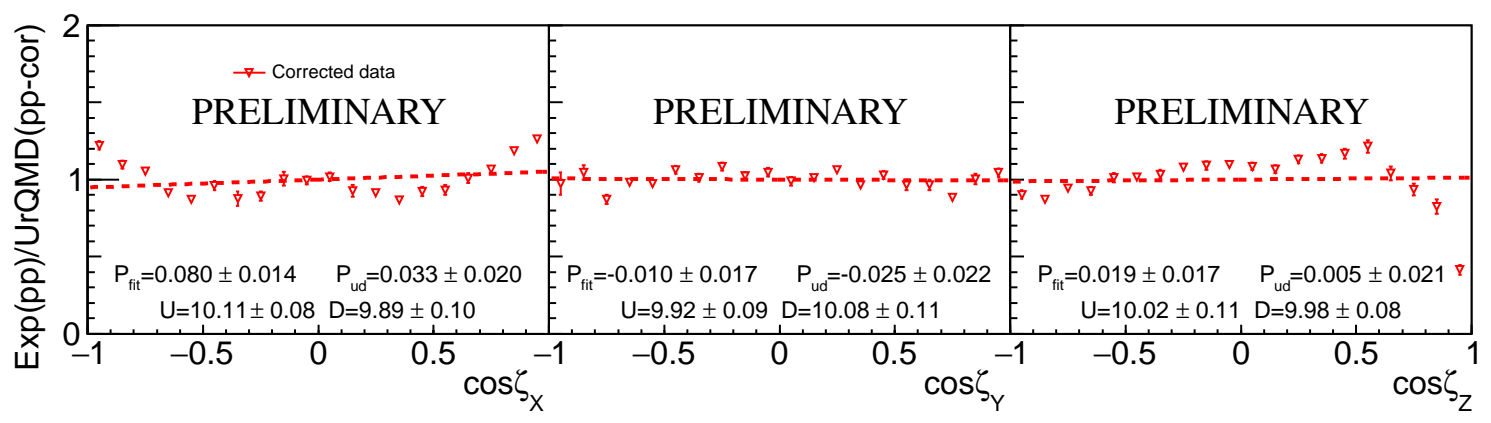

Figure 2: Ratio of the $\Lambda$ yield and the correction distribution. 


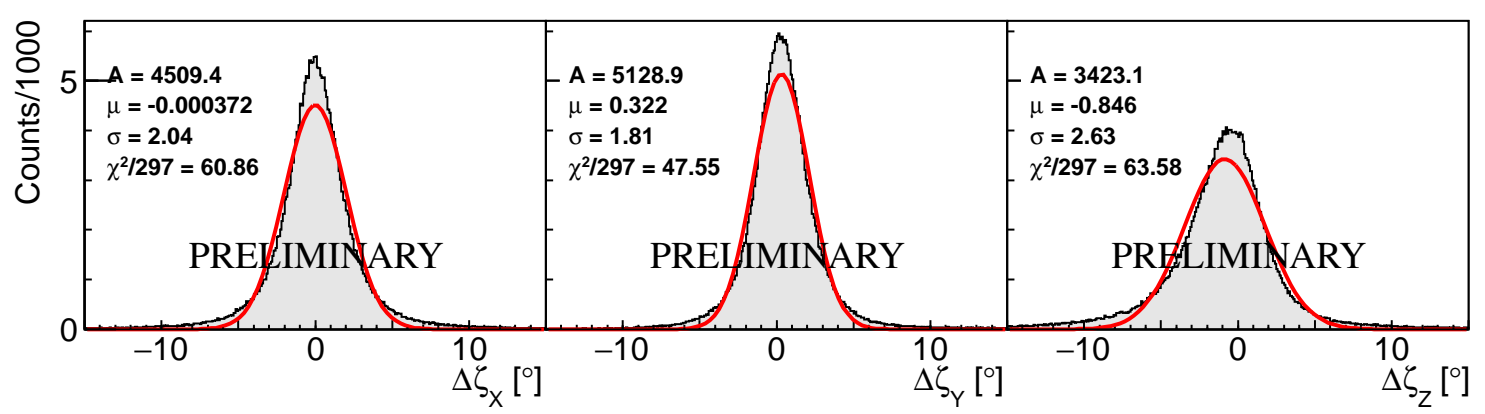

Figure 3: Difference between reconstructed and simulated polarization angle.

Table 1: Precision in the polarization angle determination for two comparable experiments at HADES.

\begin{tabular}{lllllll}
\hline Reaction & \multicolumn{2}{c}{$\Delta \cos \zeta_{x}$} & \multicolumn{2}{c}{$\Delta \cos \zeta_{y}$} & \multicolumn{2}{c}{$\Delta \cos \zeta_{z}$} \\
& \multicolumn{1}{c}{$\mu_{x}$} & $\sigma_{x}$ & $\mu_{y}$ & $\sigma_{y}$ & $\mu_{z}$ & $\sigma_{z}$ \\
\hline $\mathrm{p}+\mathrm{p}$ & -0.000372 & 2.04 & 0.322 & 1.81 & -0.846 & 2.63 \\
$\mathrm{p}+\mathrm{Nb}$ & -0.00278 & 1.93 & 0.073 & 1.68 & -0.2 & 3.17 \\
\hline
\end{tabular}

reconstructed angle has been compared with the ideal simulated value. The distribution of the differences is shown in Fig. 3. These values were directly compared with the values [11] obtained in the $\mathrm{p}+\mathrm{Nb}$ experiment at HADES [8], utilizing the same detector configuration and tracking code. The comparison is given in Table 1. While reconstruction in $x$ and $y$ directions shows good centering of the distribution, the $z$ direction has significant shift in the negative direction and the distribution is also wider. This is a result of the tracking resolution and the influence on the obtained results is currently studied. The shift is considered as a major contribution to the unexpected slope in the $\zeta_{z}$ angle in Fig. 2.

Direct comparison of the $\cos \zeta_{z}$ of the simulated and reconstructed $\Lambda$ (Fig. 4 left) shows that indeed for the higher cosine value the reconstructed yield is higher than for ideal simulation, whereas in the lower cosine range, beside very low cosine values $\left(\cos \zeta_{z}<-0.75\right)$, the situation is opposite. This effect is not very big and it could be caused by slightly improper boost calculation for the
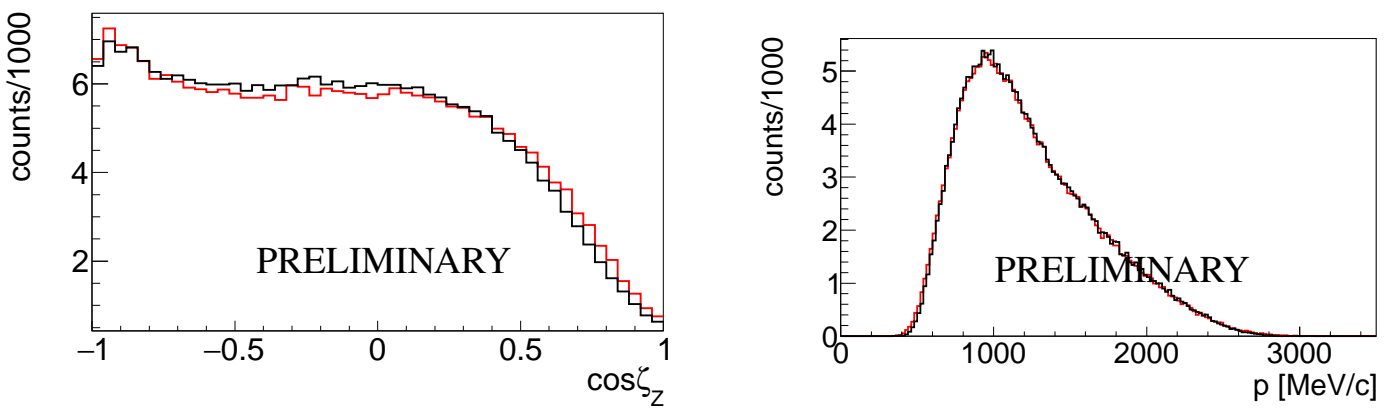

Figure 4: Simulated (black) and reconstructed (red) polarization $\zeta_{z}$ angle (left) and momentum (right) distributions of $\Lambda$ in the UrQMD simulations. 
proton, however comparison of simulated and reconstructed momentum (Fig. 4 right) shows that the momentum is reconstructed properly.

\section{Summary}

The first results of the $\Lambda$ polarization shows large uncertainty in the hyperon direction. At the moment, the influence of the simulation into these results is studied. At the same time and unexpectedly, the polarization in the spin-aligned direction $x$ shows very small positive value. Since the three polarization angles are not independent, solving the problem of the longitudinal direction should modify the distributions in the two other angles. It is then expected that the polarization in the $x$-direction will turn negative and stays consistent both with the theory predictions and the comparable experimental measurements at HADES.

\section{References}

[1] G. Bunce et al. PRL 36 (19 1976), pp. 1113-1116. DoI: 10.1103 / PhysRevLett. 36 . 1113.

[2] E.J. Ramberg et al. PLB 338.2 (1994), pp. 403-408. DOI: $10.1016 / 0370-2693$ (94) 91397-8.

[3] I. Abt et al. PLB 638.5 (2006), pp. 415-421. DoI: $10.1016 / j$. physletb. 2006.05. 040 .

[4] Jacques Soffer and Nils A. Törnqvist. PRL 68 (7 1992), pp. 907-910. DOI: 10.1103 / PhysRevLett. 68.907.

[5] S. M. Troshin and N. E. Tyurin. PRD 55 (3 1997), pp. 1265-1272. DOI: 10.1103 / PhysRevD.55.1265.

[6] J. Ellis, A. Kotzinian, and D.V. Naumov. EPJ C25.4 (2002), pp. 603-613. DOI: 10.1140 / epjc/s2002-01025-2.

[7] K. A. Olive et al. Chin. Phys. C 38 (2014), p. 090001. DOI: 10.1088/1674-1137/38/ 9/090001.

[8] G. Agakishiev et al. EPJ A50 (2014), p. 81. DOI: $10.1140 /$ epja/i2014-14081-2.

[9] G. Agakishiev et al. EPJ A41 (2009), pp. 243-277. DOI: 10 . $1140 /$ epja/i200910807-5.

[10] S.A. Bass et al. Progress in Particle and Nuclear Physics 41 (1998), pp. 255-369. DOI: $10.1016 /$ s0146-6410(98)00058-1.

[11] Christian Wendisch. "Hyperon-Produktion und -Polarisation in der Reaktion p $(3,5 \mathrm{GeV})+$ Nb mit HADES”. PhD thesis. Technische Universität Dresden, 2014. 